\title{
Nucleus subthalamicus ist langfristig der bessere THS-Zielpunkt
}

Fragestellung: Die fortgeschrittene Parkinson-Erkrankung kann durch die tiefe Hirnstimulation (THS) mit Platzierung der Elektroden im Nucleus subthalamicus (STN) oder im Globus pallidum internum (GPi) bilateral behandelt werden. Über die Langzeitwirkung auf motorische Leistung, Kognition, Stimmung und Verhalten ist wenig bekannt.

Hintergrund: Die NSTAPS-Studie - über die hier berichtet wird - hat Patienten mit einer fortgeschrittenen Parkinson-Erkrankung und behindernden Fluktuationen trotz optimaler medikamentöser Therapie zu STN- oder GPi-THS randomisiert. Die verblindeten 1-Jahres-Ergebnisse dieser Studie wurden bereits 2013 publiziert und haben einen Vorteil für die STN-THS gezeigt [1].

Odekerken VJ, Boel JA, Schmand BA et al. GPi vs STN deep brain stimulation for Parkinson disease: Three-year follow-up. Neurology 2016; 86: $755-61$
Patienten und Methoden: 90 der ursprünglich $128 \mathrm{~Pa}$ tienten konnten nach drei Jahren nachuntersucht werden. Es wurden neben der UPDRS und allgemein verwendeten Skalen ein lokal validierter kombinierter Score mit Subskalen für neuropsychologische Funktionen, Berufstätigkeit, Verlust sozialer Bindungen, Psychose, Depression und Angst eingesetzt.

Ergebnisse: Die motorische UPDRS-Skala war im Zustand ohne Medikation für die STN-Gruppe leicht aber signifikant besser (median interquartile range (IQR) nach drei Jahren, GPi 33 [23-41], STN 28 [20 - 36], p<0,04). Die Behinderung war ebenfalls geringer für die STN-Gruppe. Im Zustand mit Medikamentenwirkung gab es keine Unterschiede. Die Scores für Kognition, Stimmung und Verhalten waren nicht unterschiedlich. In der GPi-Gruppe wurden acht Patienten wegen unbefriedigender Wirkung im STN reoperiert. In umgekehrter Richtung wurde nur ein Patient reoperiert. Die Tagesdosis an L-Dopa-Äquivalenten war in der STN-Gruppe deutlich niedriger.

Schlussfolgerungen: Auch nach drei Jahren ist die STN-Stimulationswirkung auf Motorik und Behinderung im Off-Zustand besser wirksam als die der GPi-Stimulation. Für die Nebenwirkung im Bereich Kognition, Verhalten und Stimmung gab es keine signifikanten Unterschiede. Somit spricht mehr für den Zielpunkt STN als für den GPi.

\section{- Kommentar von Günther Deuschl, Kiel}

\section{Ein Register für alle operierten Patienten wäre sinnvoll}

Es gibt nur zwei große randomisierte Studien, die STN- und GPi-Stimulation vergleichen, wobei die vorliegende niederländische Studie [1] vom Studiendesign und der Durchführung her deutlich besser ist. Die amerikanische Studie [2] hat in beiden Behandlungsarmen weit unterdurchschnittliche Wirkungen auf die Motorik gezeigt, weshalb Unterschiede nicht nachweisbar waren. Die Gründe hierfür sind heftig diskutiert worden [3]. Unterschiede fehlten auch in der 3-Jahres-Nachbeobachtung [4]. Das niederländische Ergebnis dagegen zeigt die zu erwartenden Unterschiede in der besseren Wirkung im Zustand ohne Medikamentenwirkung. Wegen dieser für den schwer fluktuierenden Patienten unerträglichen Situation lässt er sich ja operieren und daher ist dies ein sehr bedeutungsvoller Outcome-Parameter. Unterschiede in den Neben- und den nicht motorischen Wirkungen haben sich auch nach drei Jahren nicht gezeigt. Auch die L-Dopa-Dosis unterscheidet sich in den Behandlungsarmen deutlich. Bemerkenswert ist überdies die Beobachtung von mehr Therapieversagern in der GPIGruppe: Tatsächliche ist der genaue Zielpunkt bei der GPi-Stimulation viel umstrittener als bei der STN-Stimulation und so wäre leicht eine größere Fehlerquote denkbar.

Insgesamt ist dies eine wichtige Studie, die an einer relativ großen Patientengruppe die Vorteile der Zielpunkte weiter zu- gunsten des STN verschoben hat und Level-II-Evidenz für die STN- und gegen die GPi-Stimulation im Regelfall erbracht hat. Es wird aber auch weiterhin die Indikation zur Stimulation des GPI geben, wenn besondere Umstände vorliegen. Da es große Studien zu diesen Fragen nicht mehr geben wird, wäre ein Register für alle operierten Patienten wichtig.

\footnotetext{
Referenzen:

1. Odekerken VJ et al. Lancet Neurol 2013; 12: $37-44$

2. Follett KA et al. N Engl J Med 2010; 362: 2077 - 91

3. Krack P et al. Nat Rev Neurol 2010; 6: 535-6

4. Weaver FM et al. Neurology 2012; 79: 55-65
}

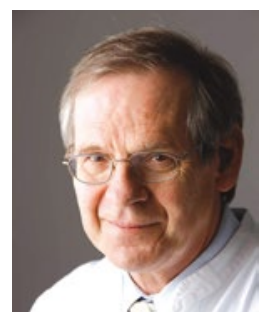

Prof. Dr. Dr. h. c. Günther Deuschl, Kiel

Emeritierter Direktor der Klinik für Neurologie, Universitätsklinikum Schleswig-Holstein, Campus Kiel (UKSH) E-Mail: g.deuschl@neurologie.uni-kiel.de 